\title{
Systemic absorption of topically applied chloramphenicol eyedrops
}

\author{
G. E. TROPE, ${ }^{1}$ J. R. LAWRENCE, ${ }^{2}$ VALERIE M. D. HIND, ${ }^{1}$ AND J. BUNNEY ${ }^{3}$ \\ From the Departments of ${ }^{1}$ Ophthalmology, ${ }^{2}$ Materia Medica, and ${ }^{3}$ Pharmacy, Stobhill Hospital, Glasgow
}

SUMMARY Five children under the age of 9 years had topical chloramphenicol drops administered 2-hourly to each eye for 5 to 7 days. Systemic absorption was not detected by 2 sensitive assays for the drug. The hazards of topical chloramphenicol and reports in the current literature are discussed.

Two cases of suspected bone marrow depression due to topical application of chloramphenicol eyedrops have been reported (Rosenthal and Blackman, 1965; Carpenter, 1975), yet there has not been a published report on the systemic absorption of this drug from the human eye. It is known, however, that topically applied chloramphenicol is absorbed into the aqueous humour in man (Beasley et al., 1975) and animals (George and Hanna, 1977); systemic absorption of the antibiotic has been detected after topical administration to the eye in animals.

\section{Methods and materials}

Five children under 9 years of age, all admitted for routine squint surgery, were given chlorampenicol topically $(0.5 \%$ chloramphenicol Redidrops) 2hourly to each eye over a 5 to 7 day period until a dose of between 40 and $52 \mathrm{mg}$ of chloramphenicol had been administered (Table 1). Urine samples were collected within 24 hours of the last dose. These samples were subjected to analysis by gasliquid chromatography (Least et al., 1977) which with thiamphenicol as internal standard had a lower limit of sensitivity of $1 \mu \mathrm{g} / \mathrm{ml}$. Polarography was also performed on these samples with a Southern Analytical Davies Diffraction Cathode Ray Oscilloscope. This method was found to be sensitive to levels of chloramphenicol from $0.05 \mu \mathrm{g} /$ $\mathrm{ml}$ upwards when aqueous standards were examined. However, because of non-specific interference from the drug-free urine the practical working limit of the assay proved to be $1 \mu \mathrm{g} / \mathrm{ml}$.

\section{Results}

These assays failed to detect any chloramphenicol in urine following the dosage schedules outlined. This

Correspondence to Dr G. E. Trope, Tennent Institute of Ophthalmology, Western Infirmary, Glasgow G11 5NT
Table 1 Patients' age and total dosage of drug applied to both eyes

\begin{tabular}{lll}
\hline Patient & Age & Total dose (mg) \\
\hline I & 3 & 48 \\
II & 8 & 52 \\
III & 2 & 47 \\
IV & 5 & 47 \\
V & 4 & 40 \\
\hline
\end{tabular}

was the case even when the extraction procedures described above were modified to allow concentration by a factor of 10:1. It thus appears that any chloramphenicol reaching the urine after ocular administration does not attain concentrations exceeding $0 \cdot 1 \mu \mathrm{g} / \mathrm{ml}$.

\section{Discussion}

The primary excretory pathway for chloramphenicol is via the kidneys; 80 to $90 \%$ of an oral dose is eliminated this way within 24 hours of administration (Goodman and Gilman, 1975). If systemic absorption of chloramphenicol is suspected from the eye, one would expect chloramphenicol to appear in the urine in higher concentrations than in the plasma. This effect has been confirmed in animal studies (Conner and Gupta, 1973). It would seem either that systemic absorption directly from the eye into the circulation or via the nasolacrimal duct and through the gastrointestinal tract does not occur in children or that urinary drug levels are below the levels detectable by apparatus at present available.

To reach a total dose of $45 \mathrm{mg}$ a patient would have to use 1 drop of chloramphenicol 3 times daily for 60 days, as 1 drop of $0.5 \%$ chloramphenicol is equivalent to $0.05 \mathrm{ml}$ and contains $0.25 \mathrm{mg}$ of chloramphenicol (Parke, Davis \& Co., personal communication). 
Two types of bone marrow effects are produced by systemic chloramphenicol administration (Editorial, 1970). Two cases have been reported implicating chloramphenicol eyedrops as the cause of the severe form of bone marrow aplasia. The report by Carpenter (1975) did not provide a definite link between this drug and the aplasia. The aplasia reported by Rosenthal and Blackman (1965), however, may well have been precipitated by chloramphenicol drops, as this patient had a strong family history of fatal aplastic anaemia precipitated by oral chloramphenicol therapy and had used topical drops for an extremely long time, namely, 2 years. In this type of case the possibility of fatal or serious results must be considered, as the likelihood of systemic absorption must be higher than in the shorter periods described above.

\section{Conclusion}

Chloramphenicol is a widely used effective topical antibiotic. Even though we were unable to detect systemic absorption of this drug after topical application to both eyes in children over a 5 to 7 day period, the possibility, however remote, still exists that systemic absorption may occur if the drug is used for excessively long periods of time. Ophthalmic practitioners should be alert to this potential hazard and its complications. Further studies to detect systemic absorption of this drug over very long periods, preferably in inflamed eyes, are indicated.

We thank Mr H. H. Fawzi for allowing us to study his patients, and Professor W. S. Foulds and Dr W. Lee for giving advice on the preparation of this paper.

\section{References}

Beasley, H., Boltralik, J. J., and Baldwin, H. A. (1975). Chloramphenicol in aqueous humour after topical application. Archives of Ophthalmology, 93, 184-185.

Carpenter, G. (1975). Chloramphenicol eye drops and marrow aplasia. Lancet, 2, 326-327.

Conner, G. H., and Gupta, B. N. (1973). Bone marrow, blood and assay levels following medication of cats with chloramphenicol ophthalmic ointment. Veterinary Medicine/Small Animal Clinic, August, 895-899.

Editorial (1970). Chloramphenicol-induced bone marrow suppression. Journal of the American Medical Association, 213, 1183-1184.

George, F. J., and Hanna, C. (1977). Ocular penetration of chloramphenicol. Archives of Ophthalmology, 95, 879-882.

Goodman, G., and Gilman, L. S. (1975). Pharmaceutical Basis for Therapeutics, 5th edn., p. 1195. Baillière Tindall: London.

Least, C. J., Wiegand, M. J., Johnson, G. F., and Solomon, H. M. (1977). Quantitative gas chromatography flame ionization method for chloramphenicol in human serum. Clinical Chemistry, 23, 220-222.

Rosenthal, R. L., and Blackman, A. (1965). Bone-marrow hypoplasia following use of chloramphenicol eye drops. Journal of the American Medical Association, 191, 136-137. 